\title{
The effects of 5-HTTLPR gene polymorphism on the behavioral reactions under emotional speech recognition among Mongolians
}

\author{
M.S. Vlasov ${ }^{1 *}$, D.V. Bazovkina3 ${ }^{3}$ S.S. Tamozhnikov², T.A. Ausheeva ${ }^{4}$, \\ A.N. Savostyanov ${ }^{2,3,4}$ \\ ${ }^{1}$ Shukshin Altai State Humanities Pedagogical University, Biysk, Russia \\ ${ }^{2}$ Institute of Physiology and Basic Medicine, Novosibirsk, Russia \\ ${ }^{3}$ Institute of Cytology and Genetics of SB RAS, Novosibirsk, Russia \\ ${ }^{4}$ Novosibirsk State University, Novosibirsk, Russia \\ *e-mail:vlasov@bigpi.biysk.ru
}

Key words: 5-HTTLPR, Speech Recognition task, Emotional intelligent

Motivation and Aim: 5-HTTLPR serotonin transporter gene polymorphism is associated with individual features in regulation of human emotional behavior. The expression of this gene was detected in the limbic structures of brain, such as amygdala. According to the most of report, SS genotype of this polymorphism is usually associated with lower level of emotional intelligent in comparison with LL and LS genotypes. However, such effects can be modulated by many other factors, including gender, age and cultural properties of people. This study comparatively investigated the effects of 5-HTTLPR polymorphism on psychological measures and behavioral reactions under recognition of emotional written sentences in the groups of Caucasoid Russians (154 persons) from Novosibirsk and Mongolian students from Khovd (54 persons).

Methods: The trait anxiety level, the neuroticism scores and the emotional intelligence scores were measured in all participants by means of different self-report inventories. Under experiments, the participants executed the error recognition task in Russian or Mongolian. 200 sentences were selected for the experiment. Half of the sentence list for each language contained a syntax error. Correct and incorrect samples were presented randomly with inter-trial interval varying between 4 and $7 \mathrm{~s}$. The subjects were instructed to judge whether a presented sample contains an error by pressing one of two buttons by dominant hand. All sentences were related with one of emotional conditions. $20 \%$ of sentences presented nonemotional description of unanimated objects, $20 \%$ of sentences described the anxiety level of participant himself, $20 \%$ of them described the anxiety level of some other persons, $20 \%$ of sentences described the aggression of participant himself and $20 \%$ described the aggression of some other persons. The participants were not instructed about emotional relation of sentences before the procedure. The speed of reaction and the quality of task execution separately for each emotional condition were used as the behavioral measures. Results: Inter-ethnic differences were detected among the Russian and Mongolian groups by comparison of behavioral reactions on syntactically correct and incorrect sentences that reflects a gramma specificity of Mongolian language. Also, the behavioral measures under recognition of sentences with aggression had some ethnic specificity. SS homozygotes showed higher scores of anxiety and neuroticism and lower scores of the emotional intelligence in comparison with the L-allele carriers among the Russian, but not for Mongolian group. In addition, the associations between 5-HTTLPR genotype, behavioral reactions on the emotionally differing sentences and the psychological measures were different among ethnic groups.

Conclusion: The effect of 5-HTTLPR polymorphism on human emotional behavior depends on the cultural parameters of participants.

Acknowledgments: This research was supported by Russian Foundation for Basic Research, project No. 16-23-03005. 